\title{
A differential analysis of sutural and remodeling growth in the human face
}

\author{
DONALD H. ENLOW, PH.D., and \\ W. STUART HUNTER, D.D.S., PH.D. \\ Ann Arbor, Miah.
}

THE purpose of the present study is to analyze and illustrate the relative contributions to over-all facial growth by (1) the sutural system and (2) the independent but closely coordinated process of bony remodeling.

It has sometimes been assumed that total increase in the size of the upper face is a combined result of two major growth processes: bone growth at sutures and bone growth along the alveolar margin and tuberosity. This is an inaccurate and ineomplete picture, since a third basic and equally significant process of facial growth is also involved. This latter process is concerned with the continuous, successive, and simultaneous remodeling growth that takes place throughout virtually all parts of the entire facial skeleton.

Remodeling is a special mechanism that provides two major and fundamental growth functions. First, as new bone is deposited on the various suture surfaces of the different facial bones, the relative positions of all regional parts of each bone are necessarily changed with respect to the new dimensions of the whole bone. Without a corresponding mechanism adapted to the simultaneous adjustment and repositioning of each regional area, the growing bone would become progressively disproportionate. The process of remodeling functions continuously to relocate individual areas and parts so that the over-all shape and proportions of the whole bone are continously maintained. This process of relocation involves sequential changes throughout the bone, so that any given part is constantly being moved by combinations of resorption and deposition on selected inner and outer cortical surfaces. For example, as the floor of the orbit is lowered in its position as a result of the increasing height of the frontal process of the maxilla during suture growth, corresponding surface deposits of new bone serve proportionately to relocate this orbital floor in a progressive upward direction, thereby constantly maintaining its position relative to the remainder of the face.

From the Departments of Anatomy and Orthodontics and the Center for Human Growth and Development, University of Michigan. 
The second function of remodeling growth in the facial skeleton is to bring about a proportionate increase in the size of all other parts of each bone as the whole bone becomes elongated at the sutures. Thus, as the frontal process of the maxilla becomes lengthened by suture deposits of new bone, the various other dimensions of this area become correspondingly and simultaneously enlarged in direct proportion. Remodeling growth is distinet from sutural growth (or condylar growth, epiphyseal growth, growth at the synchondroses, ete) but functions in direct coordination with it.

It has been shown that sutures, although representing an integral part of the total facial growth mechanism, do not in themselves provide the primary stimulus that leads to displacement and to new bone deposition., ${ }^{12}$ Rather, enclosed or adjacent soft tissues, such as the growing brain within the calvarium and the expanding cartilaginous nasal septum in the face, serve to separate the contact surfaces of individual sutures and at the same time to stimulate new bone deposition on these surfaces. Thus, bone growth at sutures is passive in nature and does not actually induce displacement but, rather, functions as a consequence of it.

\section{PROCEDURES}

Serial cephalograms of ten normal Caucasian children (five boys and five girls) at 6,9 , and 15 years of age were selected from the files of the University Elementary School Growth Study. Measurements of key anatomic dimensions were made at each age level for all individuals, corrected for cephalometric enlargement, and averaged (Table I). These values were then used in the construction of frontal and lateral composite profiles for each of the three age levels (Figs. 1 and 4).

Sutural growth calculations were based on previous findings of Björk, ${ }^{4}$ who used implant markers in growing children and found that displacement associated directly with sutural growth proceeds in an approximate 1.0 to 0.9 average ratio to bone growth along the alveolar margin at the level of the first molar. Björk's information was obtained for an age range similar to the 6- to 9-year

Table I. Average serial values* derived from ten children, equally divided as to $\operatorname{sex}$

\begin{tabular}{|c|c|c|c|c|c|c|}
\hline $\begin{array}{c}\text { Age } \\
\text { (years) }\end{array}$ & $\begin{array}{c}\text { Fronto- } \\
\text { maxillary } \\
\text { suture } \\
\text { perpendicular } \\
\text { to palatal } \\
\text { plane } \\
\text { (mm.) }\end{array}$ & $\begin{array}{c}\text { Palatal } \\
\text { plane to } \\
\text { alveolar } \\
\text { margin } \\
\text { (incisor) } \\
\text { (mm.) }\end{array}$ & $\begin{array}{c}\text { Fronto- } \\
\text { maxillary } \\
\text { suture to } \\
\text { alveolar } \\
\text { margin } \\
\text { (total at } \\
\text { incisor) } \\
\text { (mm.) }\end{array}$ & $\begin{array}{c}\text { Malar } \\
\text { width (at } \\
\text { canthus) } \\
(\text { mm.) }\end{array}$ & $\left\{\begin{array}{c}\text { Malar } \\
\text { height } \\
\text { (F-Z suture } \\
\text { to key } \\
\text { ridge) } \\
(m m .)\end{array}\right.$ & $\begin{array}{c}\text { Ptm to A } \\
\text { (parallel to } \\
\text { palatal } \\
\text { plane) } \\
\text { (mm.) }\end{array}$ \\
\hline 6 & 34.6 & 14.0 & 48.6 & 7.3 & 33.7 & 41.3 \\
\hline 9 & 37.9 & 13.9 & 51.9 & 7.1 & 36.2 & 43.5 \\
\hline 15 & 43.8 & 16.9 & 60.7 & 7.6 & 40.7 & 47.3 \\
\hline
\end{tabular}

*All values expressed in millimeters, corrected for cephalometric enlargement. 
span employed in the present study. He also found continued and proportionate sutural growth in the 15-year-old individual whom he studied.

In the size increase from the 6- to 9-year profiles, total downward movement (sutural and alveolar growth combined) at the level of the first molar was $3.9 \mathrm{~mm}$. Using Björk's implant study ratio, we determined that of this total value a $2.8 \mathrm{~mm}$. increase was a probable result of sutural activity $(1.4 \mathrm{~mm}$. on each side of the suture line) and a $2.5 \mathrm{~mm}$. increase was a result of bone deposition along the alveolar margin. For the 6- to 15-year span, the total downward movement was $19.0 \mathrm{~mm}$. at the level of the first molar. Of this total, $10 \mathrm{~mm}$. ( $5 \mathrm{~mm}$. on each side of the suture line) was a result of sutural activity, and $9 \mathrm{~mm}$. was produced by alveolar bone growth, as determined on the basis of Björk's implant data.

Equal increments on both sides of suture lines are used in the present work, since average values are employed and since previous studies have shown no major differences in growth additions on either side of the particular sutures considered here. The implant studies by Gans and Sarnat, ${ }^{5}$ in which rhesus monkeys were used, indicate that increments on both sides of sutures are approximately equal, although slight variations may occur.

On contact surfaces at the frontomaxillary and frontozygomatic sutures of the 6-year profile, $1.4 \mathrm{~mm}$. was added for the 6- to 9-year age span, and $5.0 \mathrm{~mm}$. was added for the 6- to 15-year age span (Figs. 2, $a$ and 3,a). Tracings of each individual bone within the facial complex were reassembled after sutural increments were thus added. The resulting profile, representing only sutural growth increases without corresponding remodeling changes and alveolar growth, was then superimposed over the 9- and 15-year profiles, respectively (Figs. 2,b and
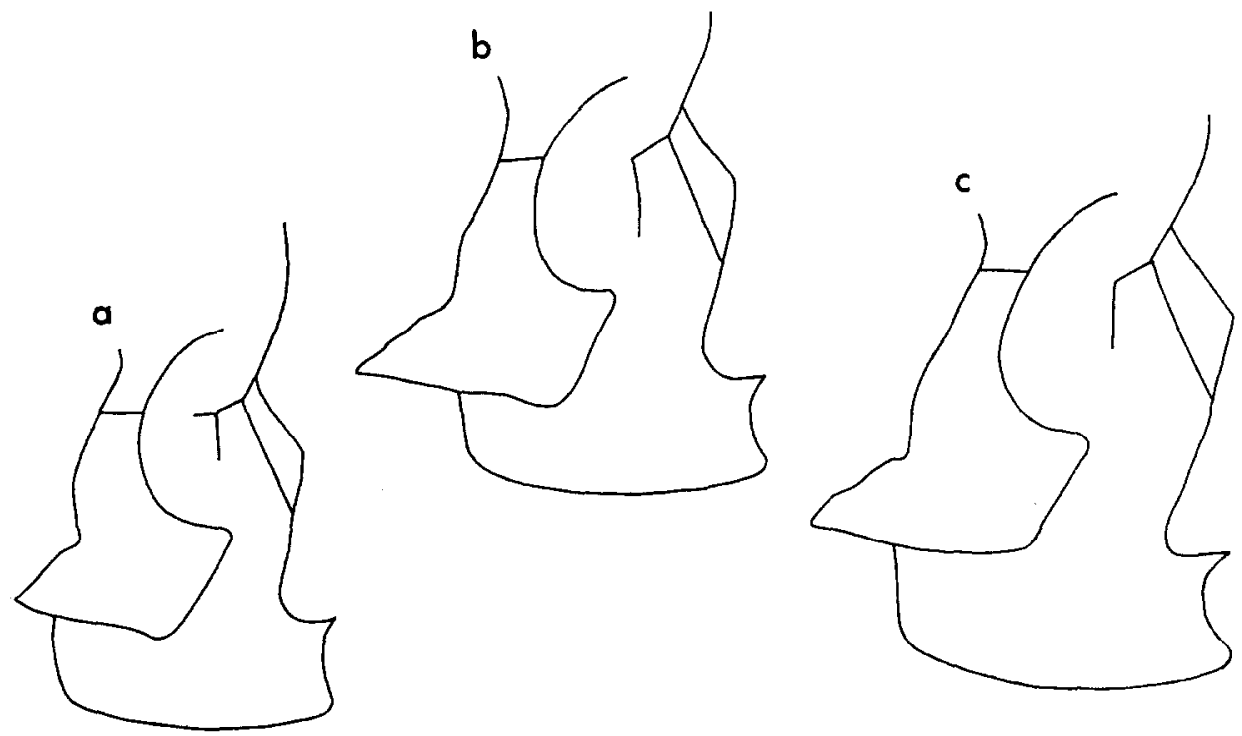

Fig. 1. Composite facial profiles at $6(a), 9(b)$, and $15(c)$ years of age. Dimensions are based on an average of measurements (Table I) taken from serial cephalograms of ten normal Caucasian children (five boys and five girls). 


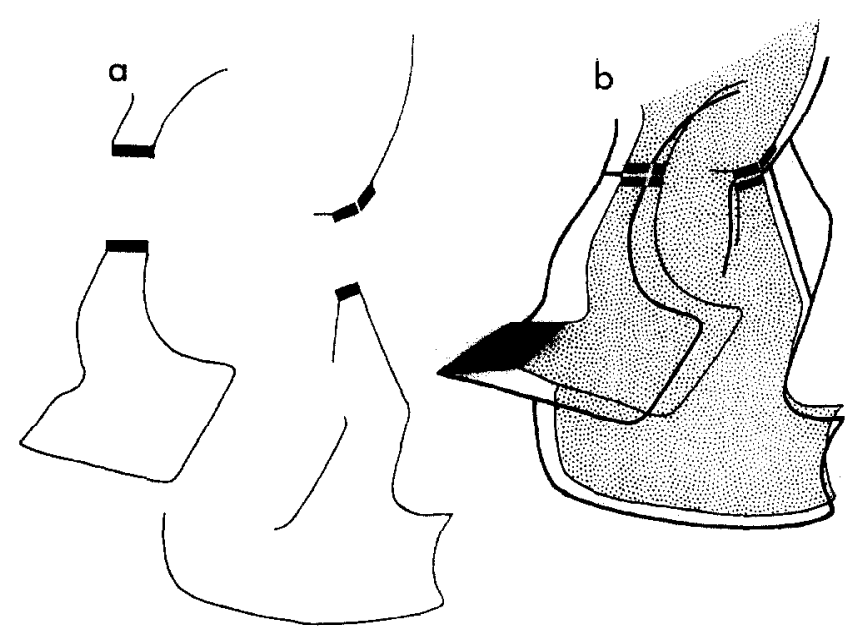

Fig. 2. Composite sutural and remodeling growth changes in profiles between the ages of 6 and 9 years. Tracings of individual component facial bones of the 6 -year profile were separated (a) and an increment of $1.4 \mathrm{~mm}$. was added to each side of the line at the frontozygomatic and frontomaxillary sutures. The parts were then reassembled and superimposed over the 9-year profile (b). Downward displacement of the entire facial complex is associated with growth at these particular sutures. Remaining differences between the facial outlines at 6 and 9 years are produced by remodeling growth which proceeds in conjunction with sutural growth.

$3, b)$. In registering all profiles, the adjusted suture lines of the 6-year facial bones were directly aligned with the corresponding suture lines of the older profiles. The resulting overlays demonstrate actual amounts of sutural growth and corresponding displacement. The differences between the lines of the younger and older profiles indicate directions and the actual extent of surface remodeling changes that accompany sutural growth increases. Growth changes in each anatomic area in the superimposed tracings give an accurate picture of the patterns of remodeling adjustments and movements, and they show the amounts of regional surface resorption or deposition involved in progressive growth.

\section{DISCUSSTON}

The special purpose of the procedure used in this study is to provide a differential analysis of (1) displacement corresponding with sutural bone growth and (2) over-all remodeling and surface growth changes that proceed in conjunction with this sutural activity. The cumulative composite of both processes is responsible for the total extent of facial growth. The superimposition of tracings using the present procedure agrees with overlays arranged according to actual known directions of remodeling growth. ${ }^{3}$

In Fig. 3, in addition to the downward and slightly forward displacement of the maxilla in association with bone deposition at the frontomaxillary suture, the nasal chamber becomes further elongated in its vertical axis by continued resorption (and endosteal bone formation) on the nasal side of the palate together with corresponding periosteal deposition on the oral side. The maxillary 


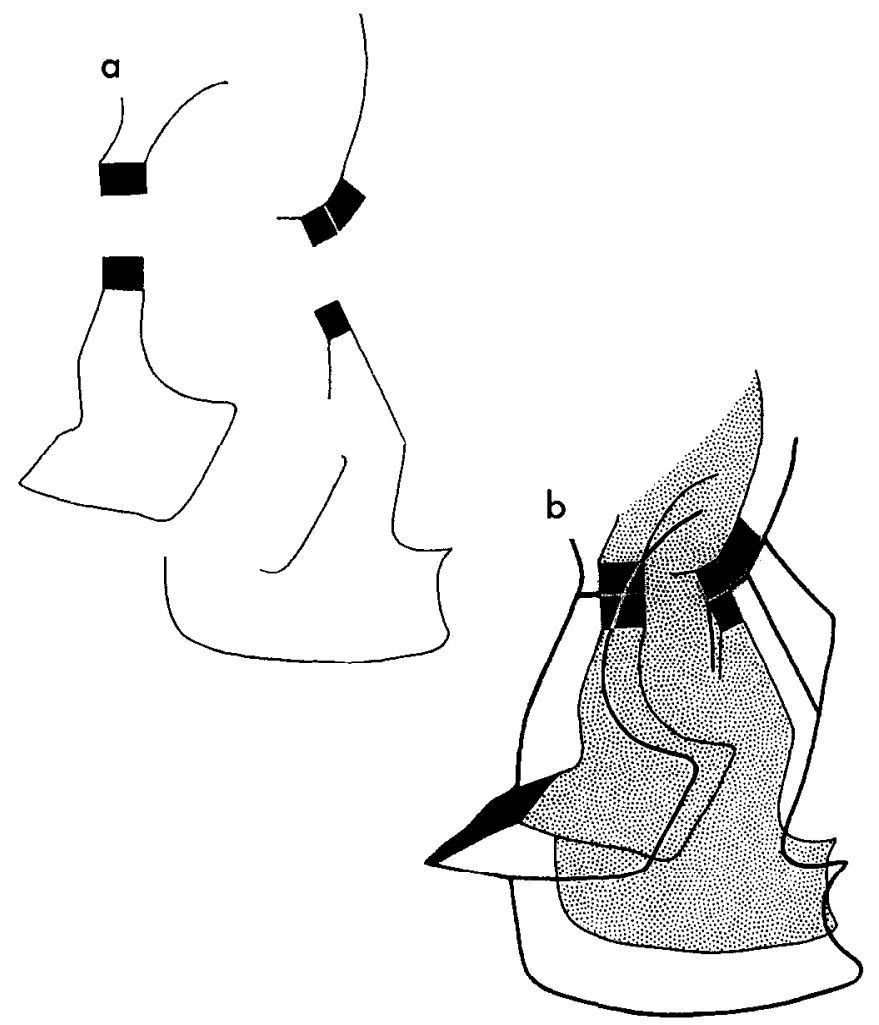

Fig. 3. Composite sutural and remodeling growth between the ages of 6 and 15 years. Tracings of individual bones of the 6-year facial complex (a) show the addition of a $5.0 \mathrm{~mm}$. increment of growth on each side of the line at the frontozygomatic and frontomaxillary sutures. The reassembled profile is then superimposed over the 15-year face, with corresponding suture lines registered (b). The differences between the profile lines at the two ages indicate the regional directions and actual extent of remodeling growth.
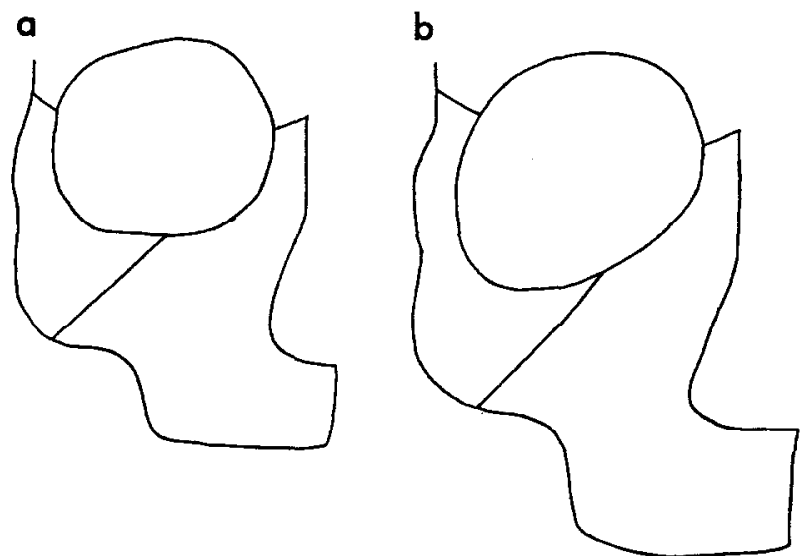

Fig. 4. Frontal outlines of the faces at 6 years $(a)$ and at 15 years $(b)$, based on average measurements of ten children. 
arch in the premaxillary region similarly brops in an almost vertical direction by progressive resorption from the entire labial surface in combination with new bone deposition on the oral sides of its cortical laminae. Alveolar depth is increased in the 6- to 15-year profiles by differentially increasing deposition along the free alveolar margin. It should be emphasized that the maxillary arehes of each successively older stage represent entircly new individual arches, each composed of new bone that had formed as the entire maxillary arch grew in an inferior direction. This inferior maxillary growth takes place in addition to the downward displacement associated with sutural bone growth. Thus, each successive age level is marked by an entirely new maxillary arch that is not only being displaced downward but actually becomes replaced by a new generation of bone and occupies a new position as it also grows in a continued inferior course. It is seen, therefore, that the alveolar bone associated with the teeth of a young growth stage is not the actual bone tissue that encloses these same teeth at a later stage.

The zygomatic bone is similarly displaced downward in conjunction with bone growth at the frontozygomatic suture. Bone resorption from the zygomatic portion of the orbital floor and massive new bone deposition along the inferior margin of the zygomatic arch serve to move and enlarge the zygoma in a continued inferior direction. Progressive resorption from the anterior face of the zygoma and the forward edge of the lateral orbital rim bring about a posterior relocation of these areas, thereby serving to maintain their relative positions with respect to the posterior-growing maxillary body.

The complex remodeling changes in the orbital and malar regions are illustrated in Fig. 5. As the maxilla moves away from the cranium in association with bone growth at the frontomaxillary suture, the medial part of the orbital floor is thereby lowered. Periosteal bone deposition on the maxillary portion of the floor of the orbit functions to raise this area in proportion, thus adjusting the level of this region to the downward positioning of the maxilla. At the same time, bone deposition on the outward-sloping orbital floor serves to move the medial orbital wall in a distinctly lateral direction. The remodeling circumstances in the zygomatic portion of the orbital floor differ markedly, however. Since this area is raised in conjunction with bone growth at the zygomaticomaxillary suture, proportionate resorption from its entire outer (periosteal) surface produces a downward relocation to keep it in line with the maxillary part of the orbit. The level of the lateral orbital rim drops with increasing age and as the entire orbit enlarges in over-all size. Such a drop is associated with the resorptive nature of this part of the orbital floor and the lateral orbital wall, both components of the separate zygomatic bone.

Marked differences in growth and remodeling occur between the upper and lower parts of the orbit. Lateral displacement of the zygoma produces bone growth at the zygomaticomaxillary suture. Note that corresponding sutural growth is not involved in the roof of the orbital cavity. The resorptive nature of endosteal bone growth in the lateral portion of the domed orbital roof is wholly responsible for the lateral enlargement of the upper orbit (Fig. 5,b), in contrast to the combined process of sutural and endosteal bone growth in the lower portion of the orbit. 
C

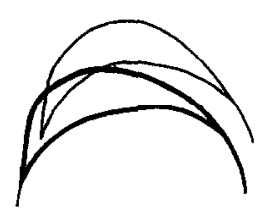

a

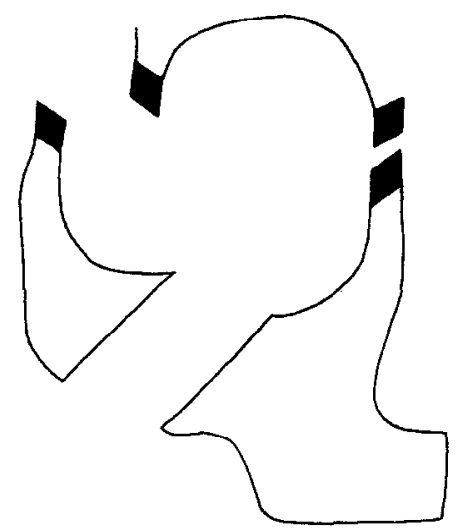

b

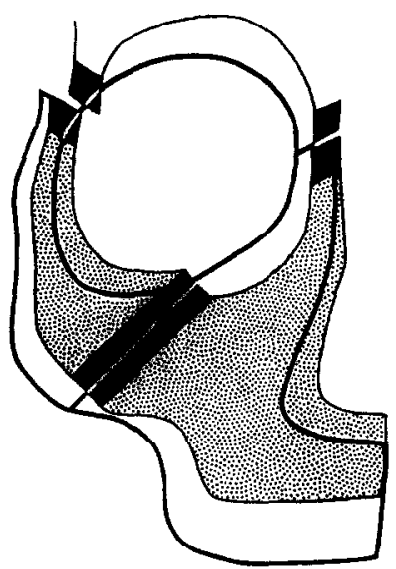

Fig. 5. Individual bones of the 6-year facial complex are separated in order to show $5 \mathrm{~mm}$. increments of new growth on each side of the suture line at the frontomaxillary and frontozygomatic sutures. The reassembled, adjusted profile is superimposed over the 15-year face, with suture lines registered $(b)$. Measurement of actual increases at the zygomaticomaxillary suture cannot be accurately indicated in this plane because of its angle. The remodeling sequence of the upper orbit is illustrated in $o$ and shows the lateral movement of the orbital roof. (See text for further descriptions.)

The resorptive nature of the lateral orbital roof, beneath the overhanging supraorbital ridge, brings about an elevation of this region as a consequence of area relocation and thereby carries the vertex of the orbital roof in a lateral as well as a forward direction (Fig. 5,c). The medial part of the roof of the orbit and the entire supraorbital rim are depository in nature. This regional remodeling pattern serves to lower the upper part of the orbit as an adjustment for new bone growth at the frontomaxillary suture and to carry the medial orbital wall in a lateral direction corresponding to a similar movement of the orbital floor. Continued bone deposition on the outer lateral surface of the zygoma and the frontal process of the zygomatic bone proceeds in conjunction with resorption (and endosteal bone formation) on the opposite inner side. This produces a lateral as well as a posterior direction of malar drift in addition to the lateral displacement of this area as a result of growth at the zygomaticomaxillary suture.

Thus, the face as a whole undergoes a process of displacement in three primary planes. First, downward thrust occurs with bone growth at the zygomaticofrontal, zygomaticomaxillary, and frontomaxillary sutures. A small amount of forward thrust of the maxilla is also related to bone growth at the latter suture. Lateral displacement is associated primarily with growth activity 
at the zygomaticomaxillary suture. Forward displacement is largely related to continued new bone growth at the zygomaticotemporal suture and, to a lesser extent, at the zygomaticomaxillary suture. Note the significantly greater degree of bone deposition at the zygomaticotemporal suture as compared with the downward-facing sutures between the frontal, maxillary, and zygomatic bones. This observation parallels the findings of Gans and Sarnat, in their implant studies on the rhesus monkey.

It is interesting to note that the actual direction of vertical remodeling growth in the maxillary arch is downward, thereby complementing downward displacement related to bone additions at the frontomaxillary and frontozygomatic sutures. The direction of horizontal growth is backward, however, in contrast to the opposite forward course of displacement associated with deposition at the zygomaticotemporal and zygomaticomaxillary sutures.

\section{SUMMARY}

As shown by bone growth at sutural contact surfaces, the particular bones involved in facial growth undergo a direct displacement away from each other. This growth process brings about a corresponding relocation of the various component parts in each individual bone. As a consequence of such relocation, remodeling adjustments take place simultaneously in order to maintain alignment, proportions, and shape, as well as to bring about compensatory increases in the over-all size of the entire bone relative to the amount of sutural bone growth. The overlay procedure used in the present study enables the worker to separate these growth processes and to visualize and demonstrate the pattern and actual extent of each.

This work was supported, in part, by United States Public Health Service Grant DE01903. The authors are indebted to Miss Rosalind Price for the illustrations used in this study. REFERENCES

1. Pritchard, J. J., Scott, J. H., and Grigis, F. G.: The Structure and Development of Cranial and Facial Sutures, J. Anat. 90: 73-86, 1956.

2. Sarnat, B. G.: Postnatal Growth of the Upper Face: Some Experimental Considerations, Angle Orthodontist 33: 139-161, 1963.

3. Enlow, D. H.: A Morphogenetic Analysis of Facial Growth, AM. J. ORThovontics 52: 283 299, 1966.

4. Björk, A.: Facial Growth in Man Studied With the Aid of Melallie Implants, Acta odont. scandinav. 13: 9-34, 1955.

5. Gans, B. J., and Sarnat, B. G.: Sutural Facial Growth of the Macaca rhesus Monkey: A Gross and Serial Roentgenographic Study by Means of Metallic Implants, AM. J. ORTHoDoNtics 37: 827-841, 1951. 